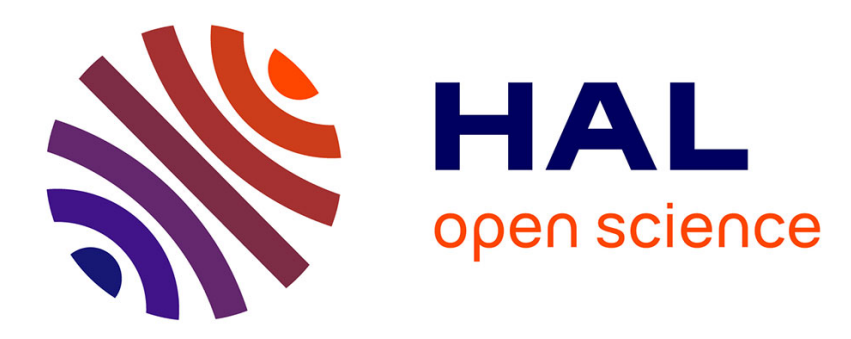

\title{
Dynamical systems with unilateral contacts
}

Christopher Glocker, F Pfeiffer

\section{To cite this version:}

Christopher Glocker, F Pfeiffer. Dynamical systems with unilateral contacts. Nonlinear Dynamics, 1992, 3, pp.245 - 259. 10.1007/BF00045484 . hal-01406288

\section{HAL Id: hal-01406288 \\ https://hal.science/hal-01406288}

Submitted on 1 Dec 2016

HAL is a multi-disciplinary open access archive for the deposit and dissemination of scientific research documents, whether they are published or not. The documents may come from teaching and research institutions in France or abroad, or from public or private research centers.
L'archive ouverte pluridisciplinaire HAL, est destinée au dépôt et à la diffusion de documents scientifiques de niveau recherche, publiés ou non, émanant des établissements d'enseignement et de recherche français ou étrangers, des laboratoires publics ou privés.

\section{(ㄷ)(1)}

Distributed under a Creative Commons Attribution| 4.0 International License 


\title{
Dynamical Systems with Unilateral Contacts
}

\author{
CH. GLOCKER and F. PFEIFFER \\ Lehrstuhl B für Mechanik, TU München, Postfach 202420, D-8000 München 2. Germany
}

\begin{abstract}
In the following a percussion drilling machine is examined as an example for mechanical systems with unilateral contacts. It is characteristic for such systems that the number of degrees of freedom changes during motion. To avoid a description of each possible system state using different sets of minimal coordinates, the constrained motion is taken into account by algebraic relations. This method has the advantage that the motion of the system and simultaneously the constraint forces are available, which is necessary to obtain conditions for a change in the state of the system. Furthermore different combinations of constraints can be easily taken into consideration in this way.
\end{abstract}

Key words: Unilateral contacts, constrained motion, friction, impacts.

\section{Introduction}

Unilateral contacts play an important role in many technical systems, above all in so called structure-variant systems, in which the number of degrees of freedom changes during the motion. Typical representatives in mechanics are all systems, whose states can be characterized by 'free flight, sliding, sticking, impact or locking', where all these states can occur in any combination. Especially the stick-slip transition contains a further interesting aspect: The friction force, representing a constraint force in the case of stiction, changes into an active force in the case of sliding friction, but depends on the passive normal force.

One possibility of dealing with such systems is to choose for all possible system states a set of minimal coordinates. But this method is to be avoided for some reasons: On the one hand a lot of state combinations results by systems which contain several structure-variant components, where for each state a set of coordinates is to be chosen and the corresponding equations of motion have to be built. On the other hand kinematical loops can arise in certain system states which make it more difficult to find appropriate coordinates and to formulate the system equations.

An effective way of treating these systems is described as follows: Starting with a description of the state which has a maximal number of degrees of freedom the constrained motion of the system is directly taken into account by algebraic equations according to the system state.

\section{Unilateral Contacts}

\subsection{Constrained Motion}

The motion behaviour of an unconstrained system is generally described by a system of differential equations 


$$
\mathbf{M}(\mathbf{q}, t) \ddot{\mathbf{q}}-\mathbf{h}(\mathbf{q}, \dot{\mathbf{q}}, t)=\mathbf{0} \in \mathbb{R}^{f}
$$

where $\mathbf{M}$ is the regular and symmetric matrix of the masses, $\mathbf{q}$ is the vector of the generalized coordinates and $\mathbf{h}$ contains all forces which act on the system. Assuming $m$ constraints $\mathbf{g}$,

$$
\mathbf{g}(\mathbf{q}, t)=\mathbf{0} \in \mathbb{R}^{m} ; \quad m \leq f
$$

the constrained motion will no longer be described by (1). To get the equations of the bound system we apply one of the principles of d'Alembert, Jourdain or Gauss, which demand that the virtual work produced by the constrained system is equal to zero,

$$
\delta \Phi^{T}(\mathbf{M} \ddot{\mathbf{q}}-\mathbf{h})=0
$$

The arbitrary displacement $\delta \Phi$ is an element of the tangent bundle of the field $\mathbf{q}$ and can be determined for example in the sense of Gauss by the second time derivative of the algebraic relation (2),

$$
\ddot{\mathbf{g}}=\mathbf{G}(\mathbf{q}, \dot{\mathbf{q}}, t) \ddot{\mathbf{q}}+\overline{\mathbf{g}}(\mathbf{q}, \dot{\mathbf{q}}, t)=\mathbf{0} ; \quad \mathbf{G} \in \mathbb{R}^{m, f},
$$

so we get with $\delta \Phi=\delta^{\prime \prime} \ddot{\mathbf{q}}$

$$
\ddot{\mathbf{g}}\left(\ddot{\mathbf{q}}+\delta^{\prime \prime} \ddot{\mathbf{q}}\right)-\ddot{\mathbf{g}}(\ddot{\mathbf{q}})=\mathbf{G} \delta^{\prime \prime} \ddot{\mathbf{q}}=\mathbf{0}
$$

Supposing the constraints are independent, rank $\mathbf{G}=m$, the permitted virtual accelerations $\delta^{\prime \prime} \ddot{\mathbf{q}}$ of the bound system evidently are perpendicular to the $m$ rows of $\mathbf{G}$. Therefore, the space of the virtual displacements is spread out by $(f-m)$ linearly independent vectors, which are perpendicular to the rows of $\mathbf{G}$. Transfering these vectors into the columns of a matrix $\mathbf{Q}$,

$$
\mathbf{G Q}=\mathbf{0} ; \quad \mathbf{Q} \in \mathbb{R}^{f, f-m},
$$

the rows of $\mathbf{G}$ together with the columns of $\mathbf{Q}$ form a basis of the $f$-dimensional space. Thus each vector is described as a linear combination of these rows and columns, for example also the whole equations of motion,

$$
(\mathbf{M} \ddot{\mathbf{q}}-\mathbf{h})=\mathbf{Q} \boldsymbol{\nu}+\mathbf{G}^{T} \boldsymbol{\lambda} ; \quad \boldsymbol{\nu} \in \mathbb{R}^{f-m} ; \quad \boldsymbol{\lambda} \in \mathbb{R}^{m}
$$

With (5), (6), (7) we can form the virtual work (3) of the system,

$$
\begin{aligned}
& \delta^{\prime \prime \mathbf{q}} \\
& \left.\Rightarrow \mathbf{Q} \boldsymbol{\nu}+\mathbf{G}^{T} \boldsymbol{\lambda}\right)=0 \\
& \Rightarrow \mathbf{Q} \boldsymbol{\nu}=\mathbf{0}
\end{aligned}
$$

Resubstituting $\mathbf{Q} \nu$ from (7) we get the equations of the constrained system,

$$
\mathbf{M} \ddot{\mathbf{q}}=\mathbf{h}+\mathbf{G}^{T} \boldsymbol{\lambda}
$$


and together with the constraints (4) a system of linear equations is available to determine the unknown $\ddot{\mathbf{q}}$ and $\boldsymbol{\lambda}$,

$$
\left(\begin{array}{cc}
\mathbf{M} & -\mathbf{G}^{T} \\
\mathbf{G} & \mathbf{0}
\end{array}\right)\left(\begin{array}{l}
\ddot{\mathbf{q}} \\
\boldsymbol{\lambda}
\end{array}\right)=\left(\begin{array}{c}
\mathbf{h} \\
-\overline{\mathbf{g}}
\end{array}\right) .
$$

Because the matrix of the masses is regular we can directly obtain the accelerations $\ddot{\mathbf{q}}$ by transforming the system equation (10) and putting them into the equations of the constraints (4),

$$
\begin{aligned}
& \mathbf{G} \mathbf{M}^{-1} \mathbf{h}+\mathbf{G} \mathbf{M}^{-1} \mathbf{G}^{T} \boldsymbol{\lambda}=-\overline{\mathbf{g}}, \\
& \Rightarrow \boldsymbol{\lambda}=-\left(\mathbf{G} \mathbf{M}^{-1} \mathbf{G}^{T}\right)^{-1}\left(\mathbf{G} \mathbf{M}^{-1} \mathbf{h}+\overline{\mathbf{g}}\right) .
\end{aligned}
$$

With $\mathbf{G}$ having full rank the matrix $\left(\mathbf{G M}^{-1} \mathbf{G}^{T}\right)$ is invertible and unique solutions for $\boldsymbol{\lambda}$ are available.

\subsection{Sliding Friction}

Sliding friction contains an interesting aspect: Two different types of forces act on the contact point of the sliding surfaces. The normal force as a passive force produces no virtual work and has no influence on the dynamics of the system. The active friction force, however, has to be taken into account in the system equations, but it depends on the passive normal force. So both of them must be available to formulate the systems equation.

First we consider the unbound system (1). Unbound means in this case that the contact condition of two surfaces that is necessary for friction is not yet taken into account. The frictionless contact of the system can be described by an algebraic equation (2) and by using its second time derivative we get the constrained system equations (4), (10), (11). The dynamical equation (10) contains the product $\mathbf{G}^{T} \boldsymbol{\lambda}$ which has the dimension of a force and represents the constraint forces of the bound system. That means that $\lambda$ is proportional to these forces, and in case of a contact of surfaces it is proportional to the normal forces. Assuming Coulomb friction the active friction forces $\mathbf{F}_{R}$ are proportional to the normal forces,

$$
\mathbf{F}_{R}=\mathbf{H} \boldsymbol{\lambda} .
$$

The matrix $\mathbf{H}$ then includes the coefficients of friction, the sign of the relative velocity in contact and other constants of proportionality. For the bound system under the influence of friction these friction forces (14) have to be taken into consideration in the equation of motion (10),

$$
\mathbf{M} \ddot{\mathbf{q}}=\mathbf{h}+\mathbf{G}^{T} \boldsymbol{\lambda}+\mathbf{H} \boldsymbol{\lambda} \text {. }
$$

Together with (4) we get two equations for the unknown $\ddot{\mathbf{q}}$ and $\lambda$,

$$
\left(\begin{array}{cc}
\boldsymbol{M} & -\left(\mathbf{G}^{T}+\mathbf{H}\right) \\
\mathbf{G} & \mathbf{0}
\end{array}\right)\left(\begin{array}{c}
\ddot{\mathbf{q}} \\
\mathbf{\lambda}
\end{array}\right)=\left(\begin{array}{c}
\mathbf{h} \\
-\overline{\mathbf{g}}
\end{array}\right) .
$$

In the same way as in (12) we get the equation for the Lagrange-multipliers, 


$$
\begin{aligned}
& \mathbf{G M}^{-1} \mathbf{h}+\mathbf{G} \mathbf{M}^{-1}\left(\mathbf{G}^{T}+\mathbf{H}\right) \boldsymbol{\lambda}=-\overline{\mathbf{g}}, \\
& \Rightarrow \boldsymbol{\lambda}=-\left(\mathbf{G} \mathbf{M}^{-1}\left(\mathbf{G} \mathbf{M}^{-1}\left(\mathbf{G}^{T}+\mathbf{H}\right)\right)\right)^{-1}\left(\mathbf{G} \mathbf{M}^{-1} \mathbf{h}+\overline{\mathbf{g}}\right),
\end{aligned}
$$

but it is remarkable that (18) generally does not produce unique solutions for $\boldsymbol{\lambda}$. Only if the matrix $\mathbf{G}$ has full rank and the matrix $\mathbf{S}:=\mathbf{G M}^{-1}\left(\mathbf{G}^{T}+\boldsymbol{H}\right)$ is invertible, we have a sufficient condition for uniqueness. If this condition is violated, some rows of $\mathbf{S}$ are linearly dependent. Transformed to upper triangular structure it will contain some zero rows. Therefore the Lagrange-multipliers together with the normal and friction forces go to infinity, which can be an indication of self locking.

\subsection{Impacts}

Another example for systems with constraints in the dynamic are collisions of bodies. Knowing the velocities $\dot{\mathbf{q}}^{-}$just before the impact, it is the aim to determine the velocities $\dot{\mathbf{q}}^{+}$shortly after the impact according to the equations of impact. In this situation the impact can be looked upon as a constraint being only active at the moment of impact. We consider the unbound dynamical system (1) that is the system in the state of free flight. The accelerations are defined by the differential quotient,

$$
\ddot{\mathbf{q}}=\frac{1}{\mathrm{~d} t}\left(\dot{\boldsymbol{q}}^{+}-\dot{\boldsymbol{q}}^{-}\right)
$$

If an impact occurs at the moment $t^{\prime}$, the change in velocities is unsteady. Therefore the accelerations are proportional to the velocity differences and go to infinity at the moment of impact,

$$
\left|\ddot{\mathbf{q}}\left(t^{\prime}\right)\right|=\infty ; \quad \ddot{\mathbf{q}}\left(t^{\prime}\right) \sim\left(\dot{\mathbf{q}}^{+}-\dot{\mathbf{q}}^{-}\right) .
$$

The equations describing the impacts are linear in the velocities before and after the impact,

$$
\mathbf{G}_{0} \dot{\mathbf{q}}^{+}+\mathbf{G}_{1} \dot{\mathbf{q}}^{-}+\overline{\mathbf{g}}_{2}=\mathbf{0}
$$

where the matrix $\mathbf{G}_{1}$ contains the coefficients of restitution. Transforming (21) by using the abbreviation

$$
\Delta \dot{\mathbf{q}}:=\left(\dot{\mathbf{q}}^{+}-\dot{\mathbf{q}}^{-}\right)
$$

we get constraints which are linear in terms proportional to the accelerations at the moment of collision,

$$
\mathbf{g}(\Delta \dot{\mathbf{q}})=\mathbf{G}_{0} \Delta \dot{\mathbf{q}}+\left(\mathbf{G}_{0}+\mathbf{G}_{1}\right) \dot{\mathbf{q}}^{-}+\overline{\mathbf{g}}_{2}=\mathbf{0}
$$

As a result the accelerations of the system go to infinity, their direction however is determined by the constraint. At the moment of impact we can split the systems equation (1), (19) into limited and impulsive terms, 


$$
\frac{1}{\mathrm{~d} t} \mathbf{M}\left(\dot{\mathbf{q}}^{+}-\dot{\mathbf{q}}^{-}\right)=\mathbf{h}_{1}+\frac{1}{\mathrm{~d} t} \mathbf{h}_{2} .
$$

Thereby all the infinite values of (24) have to be balanced. With (22) we get

$$
\mathbf{M} \Delta \dot{\mathbf{q}}=\mathbf{h}_{2} .
$$

Now the system equation (25) and the constraints (23) are available in standard form and we can use (11) to solve the problem,

$$
\left(\begin{array}{cc}
\mathbf{M} & -\mathbf{G}_{0}^{\mathrm{T}} \\
\mathbf{G}_{0} & \mathbf{0}
\end{array}\right)\left(\begin{array}{c}
\Delta \dot{\mathbf{q}} \\
\boldsymbol{\lambda}
\end{array}\right)=\left(\begin{array}{c}
\mathbf{h}_{2} \\
-\left(\mathbf{G}_{0}+\mathbf{G}_{1}\right) \dot{\mathbf{q}}^{-}-\overline{\mathbf{g}}_{2}
\end{array}\right) .
$$

By resubstituting $\dot{\mathbf{q}}^{+}$from (22) we achieve the linear system of equations for the unknowns $\dot{\mathbf{q}}^{+}$and $\lambda$,

$$
\left(\begin{array}{cc}
\mathbf{M} & -\mathbf{G}_{0}^{T} \\
\mathbf{G}_{0} & \mathbf{0}
\end{array}\right)\left(\begin{array}{c}
\dot{\mathbf{q}}^{+} \\
\boldsymbol{\lambda}
\end{array}\right)=\left(\begin{array}{c}
\mathbf{h}_{2}+\mathbf{M}^{-} \\
-\mathbf{G}_{1} \dot{\mathbf{q}}^{-}-\overline{\mathbf{q}}_{2}
\end{array}\right)
$$

where $\boldsymbol{\lambda}$ describes the impulses occuring at the impact.

\subsection{Transitions}

Generally, dynamical systems contain several structure-variant components. Each of them can be described by one constraint $g_{i}=0$. If we have $m$ components in the system, there will be altogether

$$
l=\sum_{k=0}^{m}\left(\begin{array}{l}
m \\
k
\end{array}\right)
$$

different states at most, which the system can assume. According to these $l$ states exactly $l$ different sets $\mathbf{g}_{j}=\mathbf{0} ; j \in\{1 \ldots l\}$ exist, which characterize each of the states uniquely. Further, the vectors of the constraints $\mathbf{g}_{j}$ might include components of various number. These vectors have to be formed in respect to the present state. Then the system can be treated with the former equations.

To be able to determine the transitions between the states, so called indicator functions $s_{i}$ are used, which correspond with the respective constraints $g_{i}$. These indicators always can be chosen in such a manner that a positive indicator denotes a passive constraint,

$$
s_{i}>0 \Rightarrow g_{i} \text { passive }
$$

In this case we mean by passive constraint one that has no effect on the system at that moment. If an indicator function $s_{i}$ becomes equal to zero, the corresponding constraint becomes active and has to be taken into consideration as an element of the vector $\mathbf{g}_{j}$.

In the case that an active constraint turns passive we analogously determine the transitions by using indicators $f_{i}$,

$$
f_{i}>0 \Rightarrow g_{i} \text { active . }
$$


With $s_{i}$ or $f_{i}$ being equal to zero we have a necessary (but not sufficient) condition of transition for the system to change into another system state.

\section{- Transition Sliding-Sticking}

Assuming sliding the relative velocity $v_{\text {rel }}$ in the contact of friction is not equal to zero. Positively defined we can use it as an indicator function,

$$
s_{i}=v_{\mathrm{rel}}>0 \Rightarrow \text { sticking is passive }
$$

The stick-slip transition only takes place when the stiction force excess becomes zero,

$$
f_{i}=\mu F_{N}-\left|F_{c}\right|>0 \Rightarrow \text { sticking is active }
$$

where $F_{N}$ denotes the normal force and $F_{C}$ is the tangential force due to the constraint in the case of stiction.

\section{- Impacts}

Impacts occur when the relative distance $x_{\text {rel }}$ between the colliding bodies becomes zero,

$$
s_{i}=x_{\mathrm{rel}}>0 \Rightarrow \text { impact is passive }
$$

For such transitions we do not need the indicator $f_{i}$ because it is known from the beginning that the impact takes place in an infinitely short moment and has ended with the evaluation of the impact equations. Nevertheless, we can formally introduce an indicator function $f_{i}$ to show that the impulse $p$ acts on the colliding bodies as a compressive force at the moment of impact,

$$
f_{i}=p>0 \Rightarrow \text { impact is active . }
$$

Tractions would be physically wrong.

\section{- Transition Freeflight-Sliding}

The transition freeflight-sliding demands an inelastic impact. Therefore the indicator describing the touch down of the two surfaces is the same as for the collisions,

$$
s_{i}=x_{\mathrm{rel}}>0 \Rightarrow \text { sliding is passive }
$$

After the inelastic impact the two surfaces are sliding on each other as long as the passive normal force $F_{N}$ acting on them is greater than zero,

$$
f_{i}=F_{N}>0 \Rightarrow \text { sliding is active . }
$$

These examples show that the indicators generally depend on displacements, velocities and forces. Thus mechanical systems show the characteristic that the indicators $s_{i}$ are of plain kinematical nature at so called unilateral contacts and contain only relative distances or velocities. In comparison, the indicators $f_{i}$ also include forces that can only effect the contacts in one direction.

$s_{i}$ : kinematical indicator,

$f_{i}$ : kinetical indicator . 
For the numerical computation of the instants of transition a method is used which integrates the system and calculates the switch points. The equations of motion are integrated on the currently valid system state, and simultaneously the corresponding set of indicator functions is checked in respect to the occurrence of zero transitions. If some indicators change their sign in an integration step, the first changing indicator is selected and its zero transition is determined by linear interpolation as precisely as desired. Then it will be examined, into which state the system changes at the switch point. In this area the integration is restarted at the switch point using the according set of constraints and the corresponding indicators. The applied algorithm is described in detail in $[8,9]$.

\section{Drilling Machine}

As an example for this theory a percussion drilling machine is presented. It was the aim of this examination to reduce the vibrations of the handle and keep the removal rate as high as possible. Here the geometrical design of the two installed cam discs has an essential influence on these quantities. Their changes, according to variations of the disc parameters, is to be examined.

\subsection{Mechanical Model}

The mechanical model shown in Figure 1 basically is composed of two parts, the percussion drilling machine and a hand-arm model taken from [7]. The drilling machine consists of three rigid bodies: The mass $m_{0}$ contains the drill and the first percussion disc, disc 0 , body 1 shows the second disc, disc 1, together with the gear box and the motor, and body 2 corresponds to the

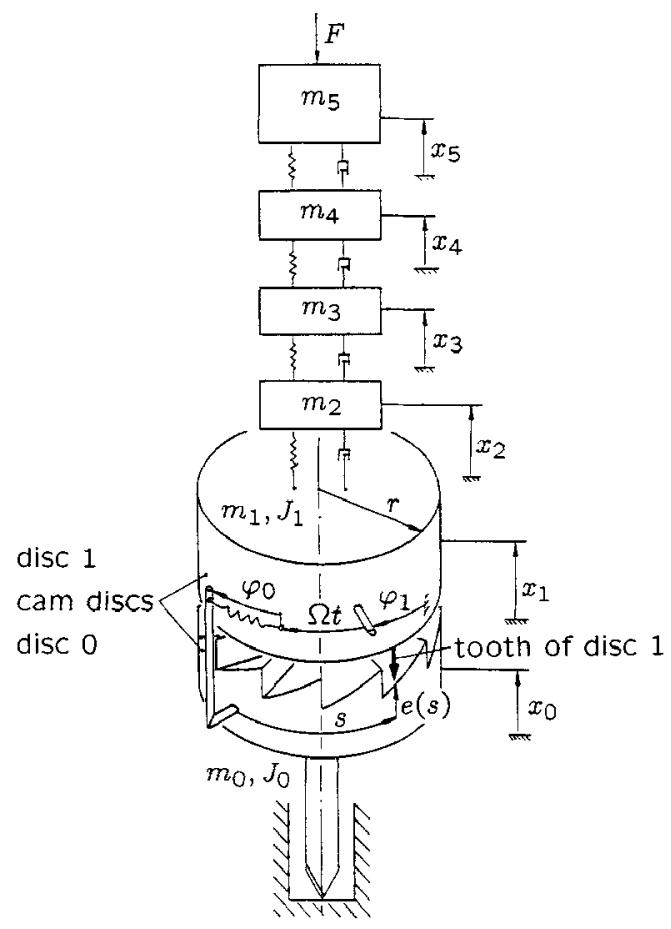

Fig. 1. Mechanical model. 
flexible mounted handle of the machine. The displacements of these three bodies are described by the absolute translational coordinates $x_{0}, x_{1}$ and $x_{2}$. The motor of the drilling machine rotates with constant angular velocity $\Omega$ and activates the drill. The elasticity of the gear unit is taken into account by the spring-damper-element $\left(c_{\varphi}, d_{\varphi}\right)$. So the drilling machine possesses two rotational degrees of freedom, where $\varphi_{1}$ describes the inertial angular displacement, and $\varphi_{0}$ denotes the relative angular displacement between motor and drill that results from the elasticity of the gear unit. The two percussion dises with radius $r$ sit on the bodies 0 and 1. Both discs hold cams with similar geometrical form, which is described by a function $e(s)$ on its outer surface. During operation the cams slip on each other until they loose contact on their tops. The following free-flight state of the drilling machine continues until the two discs hit each other again because of the pressing force $F$. The resulting impact causes the drilling feed.

To be able to describe the contour of the cams we have to find a function $e(s)$ which is continuous in its second derivative. This has to be demanded in order to avoid jumps in the constraint normal force during the sliding of the cams. In Figure 2 a function $f(x)$ is plotted,

$$
f(x)=c x\left(\frac{x}{x-b}\right)^{a}
$$

which fulfills this demand. Using some linear transformations

$$
f(x) \rightarrow g(x) \rightarrow h(x) \rightarrow e(s)
$$

we fit $f(x)$ to the searched outline $e(s)$. The three parameters $a, b$ and $c$ in (37) can be used to determine the length of the cams $l$, the angle of inclination $\alpha$ and the bend radius $R$ at the top of the cams. The variable $s$ depends on the generalized coordinate $\varphi_{0}$ and explicitly on the time $t$ due to the rotation of the motor, see Figure 1,

$$
s\left(\varphi_{0}, \Omega t\right)=r\left(\Omega t+\varphi_{0}-k\right) ; \quad k=\text { const. }
$$

that means that $e$ is nonlinear in $\varphi_{o}$ and $t$.
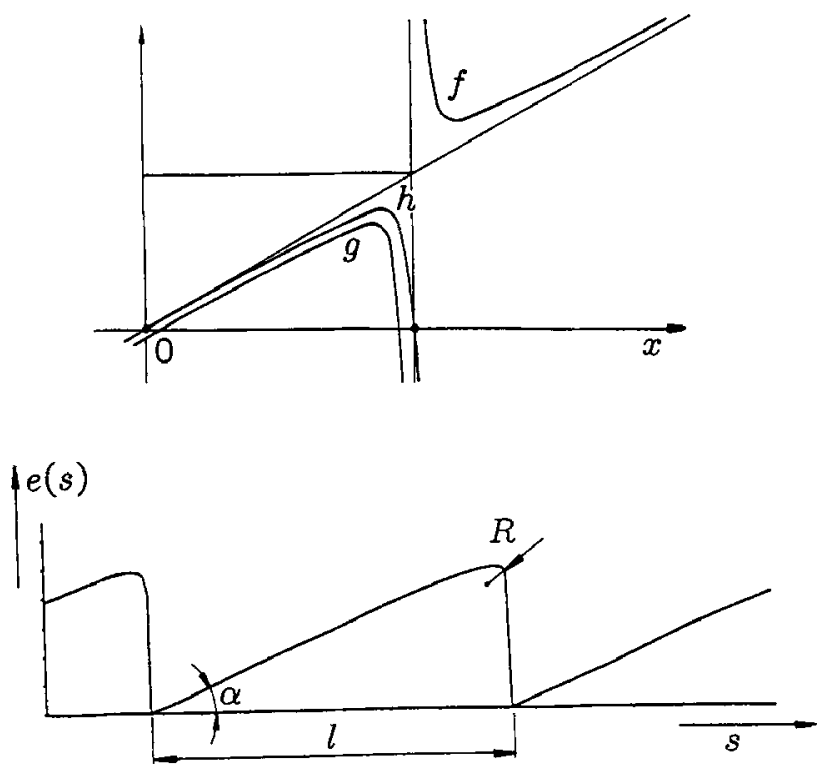

Fig. 2. Contour function. 
To be able to compute the motion of the system we have to determine the equations of motion for each system state. In this case three different states are to be distinguished:

- The free-flight state means that there is no contact between the two cam discs.

- An impact occurs when the two cam discs hit each other.

- After the impact the cams of the discs slide on one another.

For the possible transitions between these states see Section 3.5, Figure 5.

\subsection{Free Flight}

During the free flight the motion of the system can easily be described by a linear differential equation system,

$$
\mathbf{M} \ddot{\mathbf{q}}+\mathbf{D} \dot{\mathbf{q}}+\mathbf{K q}=\mathbf{h} \in \mathbb{R}^{8},
$$

where $\mathbf{M}$ is the matrix of the masses, $\mathbf{D}$ contains the coefficients of damping and $\mathbf{K}$ those of stiffness. The vector $\mathbf{h}$ includes the pressing force, and $\mathbf{q}$ are the minimal coordinates describing the free flight state of the system,

$$
\mathbf{q}^{T}=\left(x_{0} x_{1} x_{2} x_{3} x_{4} x_{5} \varphi_{0} \varphi_{1}\right) .
$$

\subsection{Impacts}

The event that occurs when the two cam discs come into contact after the free flight state is modeled by two inelastic impacts in succession. During the first collision cam disc 1 hits disc 0 , which stands idle in axial direction, see Figure 3. An inelastic direct impact takes place between the discs. To describe this impact we introduce coordinates $\xi$ and $\eta$ in such a way that the absolute velocities $\dot{\xi}$ are perpendicular to the outline of the cams in the contact point. The inclination of the
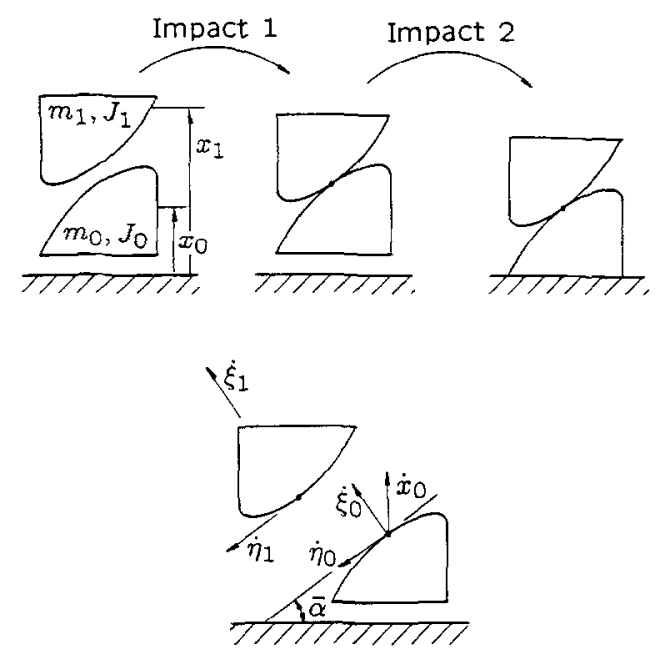

Fig. 3. Impacts. 
tangent line is determined by the first derivative of the contour function $e$,

$$
\tan \bar{\alpha}=e^{\prime}(s)
$$

So a linear transformation from the coordinates of free flight $\dot{\mathbf{q}}$, see (41) to the impact coordinates $\dot{\tilde{\mathbf{q}}}$ is available,

$$
\dot{\mathbf{q}} \rightarrow \dot{\tilde{\mathbf{q}}}=\left(\dot{\xi}_{0} \dot{\xi}_{1} \dot{\eta}_{0} \dot{\eta}_{1}\right)^{T}
$$

We consider the first inelastic impact in such a way that the relative velocity of the cams in $\xi$-direction disappears shortly after impact. With the chosen set of coordinates (43) the constraint is known,

$$
\dot{\xi}_{0}^{+}-\dot{\xi}_{1}^{+}=0
$$

and the terms in (21) are determined,

$$
\mathbf{G}_{0}=(1-100) ; \quad \mathbf{G}_{1}=\mathbf{0} ; \quad \mathbf{g}_{2}=\mathbf{0}
$$

Now we can use (27) to determine the velocities after the first impact. Both discs are now in contact and are moving in axial direction towards the bottom of the drill hole. There the second impact takes place. The velocities shortly after the first impact and just before the second are the same. Again the second impact is modeled inelastically, so we can formulate the constraints: The two cam discs will not lose contact after this impact,

$$
\dot{\xi}_{0}^{+}-\dot{\xi}_{1}^{+}=0
$$

and the drill has to stay at the bottom of the drill hole,

$$
\dot{x}_{0}^{+}=0 ; \Rightarrow \dot{\xi}_{0}^{+} \cos \bar{\alpha}-\dot{\eta}_{0}^{+} \sin \bar{\alpha}=0 .
$$

As a result we get the magnitudes of (21) describing the constraints of this impact,

$$
\begin{aligned}
& \mathbf{G}_{0}=\left(\begin{array}{cccc}
1 & -1 & 0 & 0 \\
\cos \bar{\alpha} & 0 & -\sin \bar{\alpha} & 0
\end{array}\right) \\
& \mathbf{G}_{1}=\mathbf{0} ; \quad \mathbf{g}_{2}=\mathbf{0} .
\end{aligned}
$$

According to the second constraint the vector of the Lagrange multipliers $\lambda$ in (27) contains in its second component the impulse $\lambda_{0}$ between the drill and the bottom. We obtain the removal rate per impact $V_{S}$ which is achieved by percussion drilling proportional to the second power of this impulse,

$$
\lambda=\left(\begin{array}{c}
\lambda_{1} \\
\lambda_{0}
\end{array}\right) ; \quad V_{S}=k \lambda_{0}^{2} ; \quad k=\text { const. }
$$

With that we attain the removal rate per time unit by averaging the impacts which take place during the simulation time. Now the velocities after the second impact are known and the state 'impact' of the system has ended. 


\subsection{Sliding}

For sliding after the impacts the normal force $F_{N}$ must be known to evaluate the sliding friction force $F_{R}$ according to Coulomb's law. $F_{N}$ is perpendicular to the cam's outline, $F_{R}$ is tangential to it (Figure 4). The equations of the unbound system are already known by the free-flight state (40), so we only have to formulate the constraint and to add the corresponding forces in the dynamical equations. The constraint (2) for contact is simple, see Figure 4,

$$
\mathbf{g}(\mathbf{q}, t)=x_{1}-e(s) ; s=s\left(\Omega t, \varphi_{0}\right)
$$

In this case the constraint is nonlinear in $\mathbf{q}$. By differentiating $\mathbf{g}$ two times in respect to time we get the constraint which is linear in $\ddot{\mathbf{q}}$,

$$
\ddot{\mathbf{q}}=\ddot{x}_{1}-e^{\prime} \ddot{s}-e^{\prime \prime} \dot{s}^{2},
$$

and the unknowns $\mathbf{G}$ and $\overline{\mathbf{g}}$ in (4) are available with (40),

$$
\begin{aligned}
& \mathbf{G}=\left(010000-e^{\prime} r 0\right), \\
& \overline{\mathbf{g}}=-e^{\prime \prime}\left(\Omega+\dot{\varphi}_{0}\right)^{2} .
\end{aligned}
$$

Now we still have to take the friction forces into account in the systems equation (40). On the one hand we get the constraint normal forces $\mathbf{f}_{n}$ by using for example Newton-Eulers method,

$$
\mathbf{f}_{n}=\left(010000-e^{\prime} r 0\right)^{T} F_{N} \cos \bar{\alpha}
$$

on the other hand we know that the vector which contains the constraint forces is described by $\mathbf{G}^{T} \boldsymbol{\lambda}$. Comparing (52) with (54) we can identify $\boldsymbol{\lambda}$ as

$$
\lambda=F_{N} \cos \bar{\alpha} .
$$

The vector of the friction forces $\mathbf{f}_{r}$ is determined by $\mathbf{H} \boldsymbol{\lambda}$. Using Coulomb friction with the coefficient of friction $\mu$ we get the friction forces $\mathbf{f}_{r}$ in the same way by Newton-Euler,

$$
\mathbf{f}_{r}=\left(0 \mu e^{\prime} 00000 \mu r 0\right)^{T} F_{N} \cos \bar{\alpha}
$$
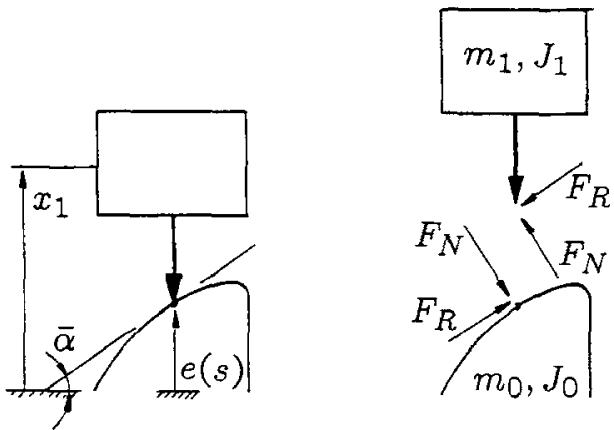

Fig. 4. Sliding: constraint and forces. 
With $\lambda$ from (55) the matrix $\mathbf{H}$ is determined,

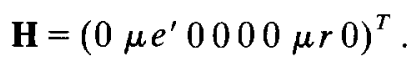

With (40), (52), (53) and (57) all terms are known to solve the linear system of equations (16) which describe the state 'sliding' of the model.

\subsection{Transitions}

At this point the indicator functions that give necessary conditions of transition between the system states have to be developed. The transition freeflight-sliding requires one kinematical and kinetical indicator with (35), (36). The kinematical indicator is obtained by the relative distance between the discs,

$$
s_{1}:=x_{1}-e(s)
$$

As a kinetical indicator we can directly use the Lagrange-multiplier $\boldsymbol{\lambda}$ from (55), because it is proportional to the normal force,

$$
f_{1}:=\boldsymbol{\lambda} \sim F_{N}
$$

Figure 5 shows all possible transitions of the system. The free-flight state $Z_{1}$ is valid as long as the distance $s_{1}$ between the discs is greater than zero. When this distance disappears, $s_{1}=0$, the two impacts $Z_{2}$ take place. Impacts lead to jumps in the velocities, therefore the kinetical indicator $f_{1}$ contains discontinuities at the moment of impact. So two transitions after the impact are possible: For normal forces less than zero, $f_{1}<0$, the system returns to the free-flight state $Z_{1}$, and for positive normal forces $f_{1}>0$, sliding $Z_{3}$ takes place. During sliding the normal force decreases continuously to zero. Then the two discs loose contact and again the state free-flight $Z_{1}$ is valid.

With these preliminary reflections all needed relations are available for integration of the system equations.

\subsection{Results}

Figure 6 shows the behaviour of the percussion drilling machine for percussion drilling in concrete. The removal rate is plotted in dependency of the rotational speed of the motor for different pressing forces. We can see that the removal rate grows with increasing speed of rotation and increasing pressing forces. This can be explained by the fact that on the one hand the percussion

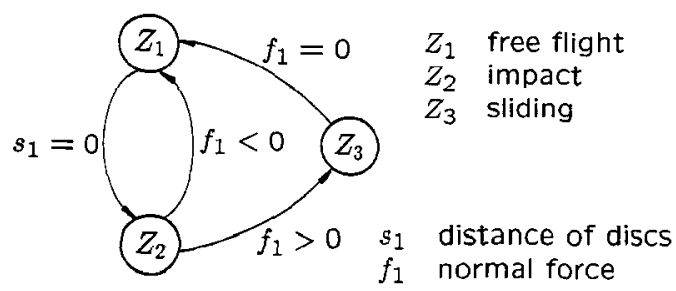

Fig. 5. Transitions. 


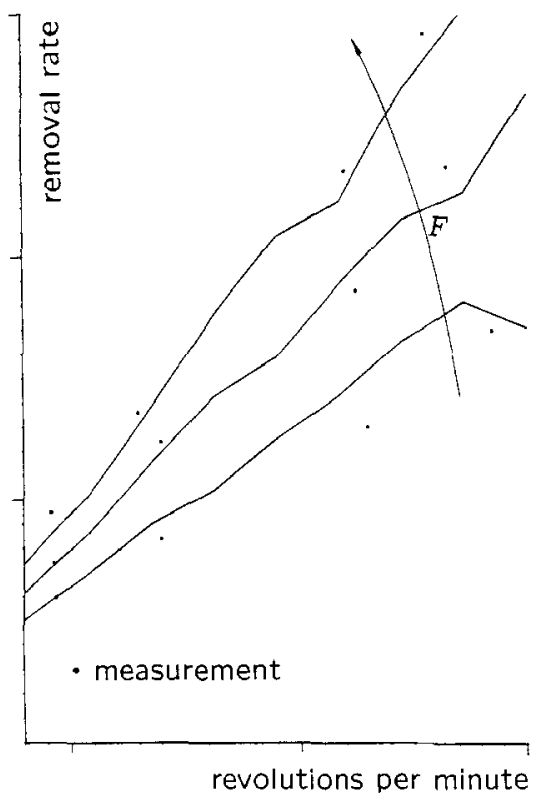

Fig. 6. Drilling in concrete.

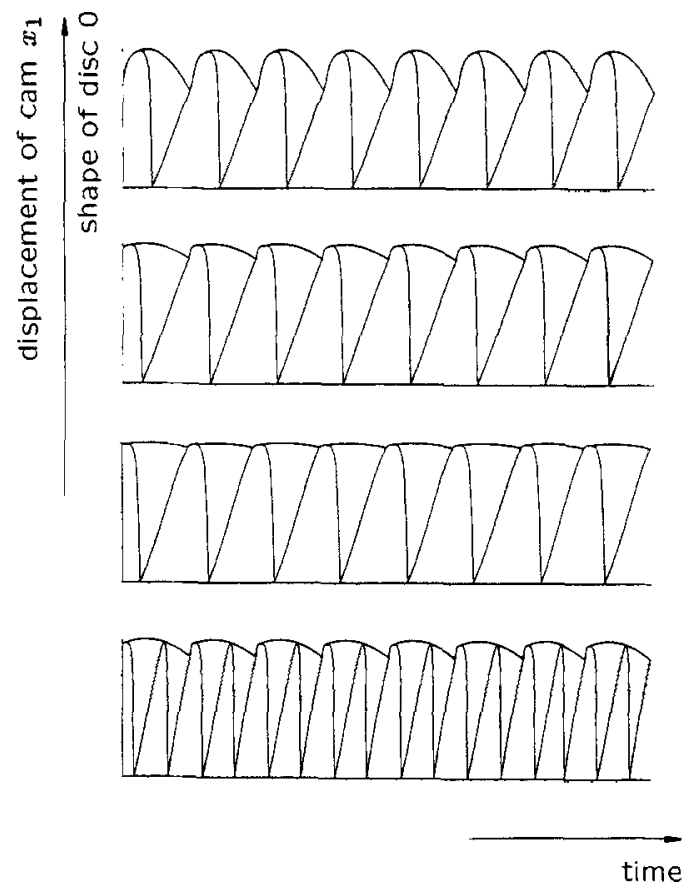

Fig. 7. Time behaviour of displacement $x_{1}$.

frequency and on the other hand the impulse transfered by the impact grows. The lines result from calculation and are confirmed by measurements, represented by the dots.

In Figure 7 the shape of disc 0 and the time behaviour of the coordinate $x_{1}$ which corresponds to the axial displacement of disc 1 is plotted for different rotational speeds. The kinks and the different smoothing out on the top of the cams result from the arising impacts and effects of dry friction. With an increasing number of revolutions the contact point of the cams moves closer to the top, until a state is reached where the cams jump from one top to the next. With a further increase of the rotational speed only every second cam is hit.

This transition is shown in the phase space portraits, Figure 8. It should be kept in mind that mechanical systems with impulsive and stick-slip processes are highly nonlinear due to unsteady transitions and a changing of degrees of freedom. Therefore, such systems may be called structure-variant. In Figure 8 the first two pictures correspond to the case that every cam of the discs is hit. It is easy to make out the sliding and the free flight state, followed by an impact. By increasing the rotational speed, picture (b), the hitting point of the cams moves upwards and the limit cycle gets smaller. The maximal altitude of $x_{1}$ in the free flight state however remains nearly constant. Further increasing of the rotational speed leads to a bifurcation of the solution. Picture (c) shows a two-periodic solution. The cams are hit at two different points which are alternately closer to the top and closer to the ground of the cams. If the rotational speed rises even more the solution bifurcates again by period doubling. Picture (d) shows a periodic solution of higher order which becomes irregular in picture (e) and changes finally in picture (f) into the solution where only every second cam is hit. The same behavior is observed at the transition from the second to the third hit cam. The aim of this examination is to analyse the influence of the geometrical design of the discs on the removal rate and handle vibrations, where the removal rate was calculated in dependency of the impulses, (49), and the handle vibrations were determined proportional to the 

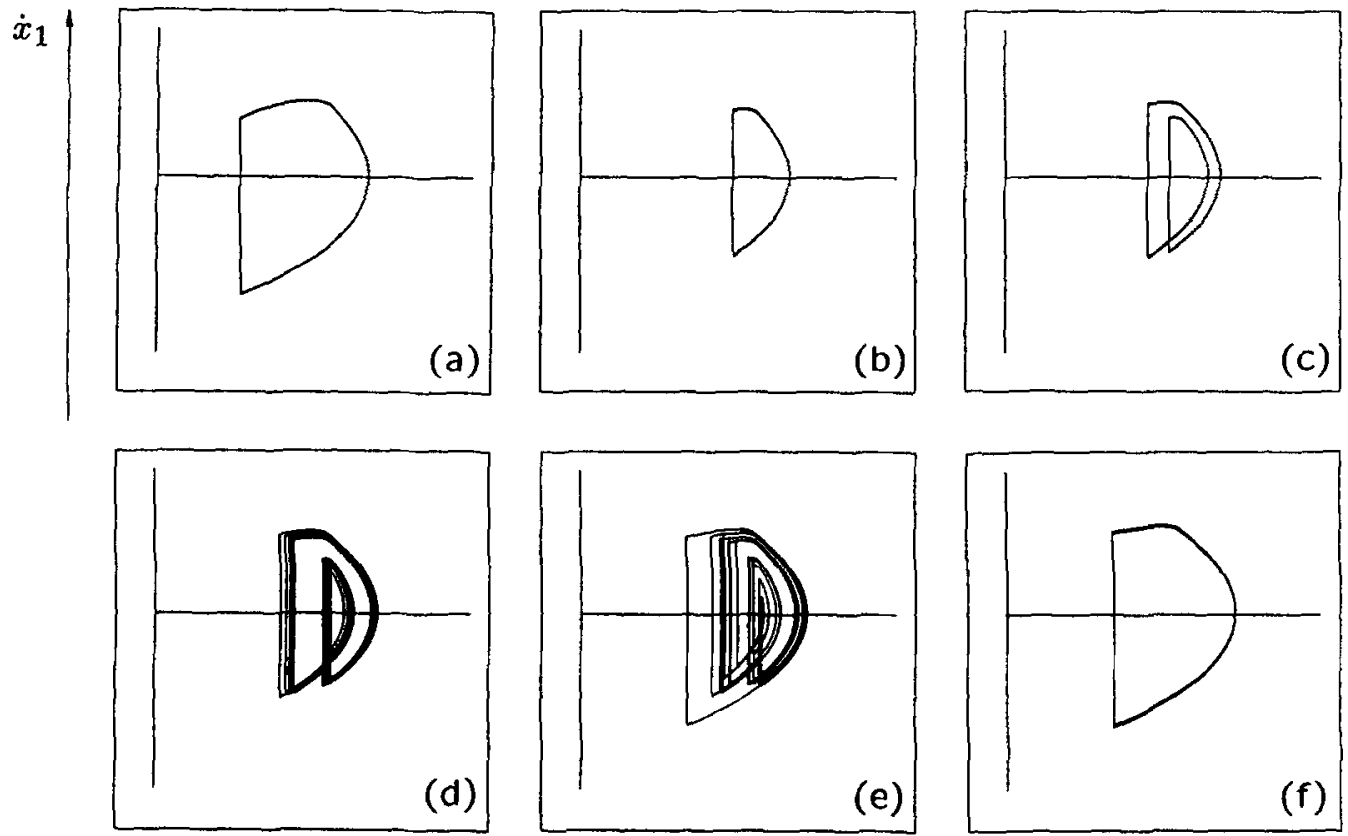

Fig. 8. Phase space portrait.

$x_{1}$
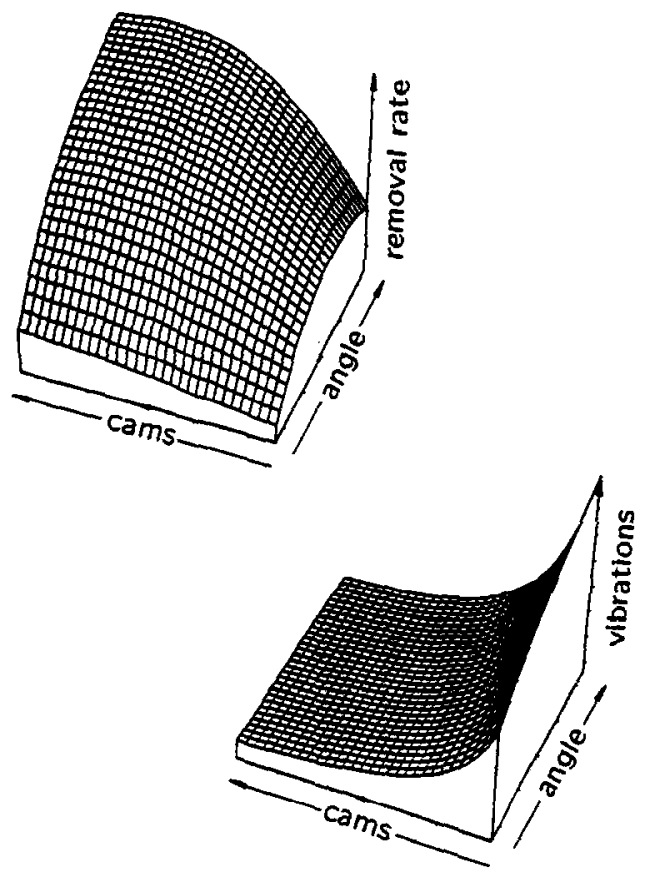

Fig. 9. Removal rate and handle vibrations. 
root-mean-square-value of the Fourier-coefficients of the handle accelerations. The angle of inclination $\alpha$ and the number of cams of one disc were chosen as design parameters. Figure 9 shows the result of this variation for parameter intervals where only that solution exists where every cam is hit. With increasing number of cams the hitting point of the cam discs moves toward the top of the cams. Thereby the frequency of impact increases and the handle amplitude decreases, which is shown by an increasing removal rate and decreasing handle vibrations. If the angle of inclination is enlarged, a more powerful impulse in axial direction takes place. The result is a better removal rate, but also slightly higher handle vibrations.

\section{References}

1. Brandl, H. and Hajek, M., 'Mechanische Systeme mit Trockenreibung', ZAMM 68, 1988, T59-T61.

2. Lötstedt, P., 'Numerical simulation of time-dependent contact and friction problems in rigid body mechanics', TRITA-NA-8214, 1982.

3. Moreau, J. J., 'Unilateral contact and dry friction in finite freedom dynamics', Non-Smooth Mechanics and Applications, CISM Courses and Lectures, Springer-Verlag, 1987.

4. Pfeiffer, F. and Kücükay, F., 'Eine erweiterte mechanische Stosstheorie und ihre Anwendung in der Getriebedynamik', VDI-Z 127, 1985, 341-349.

5. Pfeiffer, F.. 'Uber unstetige, insbesondere stosserregte Schwingungen', Z. Flugwiss. Weltraumforsch., 1988, 12358367.

6. Pfeiffer, F., 'Dynamical systems with time-varying or unsteady structure', $2 A M M 71,1991$, T6-T22.

7. Ueltzer, G., Uelzig-Thiel, R., and Schatte, M., 'Ein mathematisches Schwingungsmodell für das menschliche Hand-Arm-System', Maschinenbautechnik 29, 1980, 54-58.

8. Hajek, M., 'Reibungsdämper für Turbinenlaufschaufeln', VDI Fortschrittberichte, Reihe 11, Nr. 128, VDI-Verlag, 1990.

9. Prestl, W., 'Zahnhämmern in Rädertrieben von Dieselmotoren', VDI Fortschrittberichte, Reihe 11, Nr. 145, VDIVerlag, 1991. 